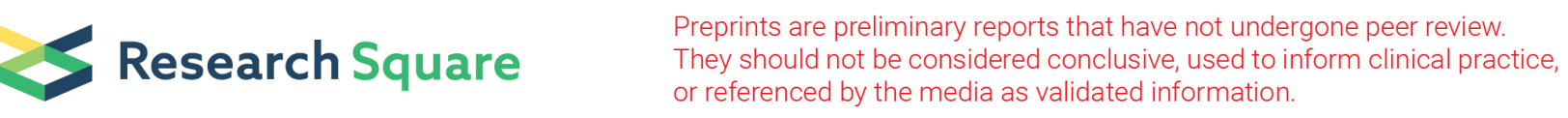

\title{
Neurological manifestations of COVID-19 associated multisystem inflammatory syndrome in adults (MIS-A)
}

Boby Varkey Maramattom ( $\square$ bobvarkey@gmail.com )

Aster Medcity https://orcid.org/0000-0001-7032-4412

\section{Research Article}

Keywords: Multisystem inflammatory syndrome in adults, neurological, India, Post vaccination MIS-A, COVID reinfection

Posted Date: July 28th, 2021

DOl: https://doi.org/10.21203/rs.3.rs-694495/v1

License: (c) (1) This work is licensed under a Creative Commons Attribution 4.0 International License.

Read Full License 


\section{Abstract}

Introduction Multi-system inflammatory syndrome (MIS) is a para infectious or post infectious extrapulmonary complication of COVID-19. Neurological complications are better described in children with Multi-system inflammatory syndrome (MIS) in children (MIS-C). There is a paucity of literature about the neurological manifestations of Multi-system inflammatory syndrome (MIS) in adults (MIS-A).

Materials and methods Over a 12-month period, from June 2020 to June 2021, over 350 patients were admitted to our medical and COVID ICUs. Of these, eight patients $(2.2 \%)$ admitted to our ICU were detected to have a neurologic MIS-A.

Results Most patients had a stroke (72.5\%). Other manifestations included transverse myelitis, COVID encephalitis, acute encephalopathy, rhabdomyolysis and critical illness myo-neuropathy.

Conclusions Most patients with MIS-A and severe neurological involvement had a poor outcome.

\section{Take Home Messages}

MIS-A can be associated with severe neurological complications.

Thrombotic microangiopathies may contribute to cerebrovascular events and should be actively looked for and managed.

\section{Introduction}

Multi-system inflammatory syndrome (MIS) is a para infectious or post infectious extra-pulmonary complication of COVID-19. Initially this syndrome was recognized in a cluster of children with severe hyperinflammatory with multi-organ dysfunction and termed Multi-system inflammatory syndrome (MIS) in children (MIS-C). These children presented with cardiogenic shock, prominent gastrointestinal and dermatological features, and elevated inflammatory markers, resembling Kawasaki disease (KD) or Toxic shock syndrome (TSS).

Since June 2020, MIS has been recognized in adults (MIS-A). Unlike COVID-19 associated hyperinflammatory syndrome (COVID-HIS), which is accompanied by respiratory failure, patients with MIS-A had minimal respiratory symptoms, hypoxemia, or pulmonary radiographic abnormalities (< $1.7 \%) .{ }^{1}$ As prominent cardiac or multi-system involvement is often used for diagnosis of MIS-As these cases are often managed in medical Intensive care units (ICU) and neurologists do not encounter them.

In both adults and children, it is becoming clearer that the spectrum of MIS-A and MIS-C varies from mild to severe variants. Patients can present with multi-organ system dysfunction without severe cardiovascular involvement. Neurological findings in MIS-A comprise of headache, encephalopathy or large vessel occlusion (LVO), and stroke. In MIS-C, approximately $27 \%$ of patients develop neurological manifestations. These include headaches, meningism/meningitis, an encephalopathy, seizures, ataxia or 
proximal muscle weakness. MIS-C patients may show transient cytotoxic changes in the corpus callosum (Boomerang sign), ischemic infarcts, intracranial hemorrhage or nonspecific T2/fluid-attenuated inversion recovery hyperintensities with restricted diffusion. ${ }^{2-4}$

As it is likely that MIS-A patients can present with neurological manifestations at onset and develop cardiac manifestations later in the illness or may lack severe cardiovascular involvement throughout the course of illness, it is necessary to define the spectrum of MIS-A We sought to determine primary neurological manifestations of MIS-A presenting to our institution in 2021.

\section{Materials And Methods}

Over a 12 month period, from June 2020 [January 2021 to June 2021], 350 patients were admitted to our institution in medical and COVID-ICUs with COVID-19 or post COVID-sequelae. Neurological MIS-A was identified from case records of primary admissions to the neurology ICU as well as through neurology consultations from the COVID-19 ICU for moderate to severe manifestations including encephalopathy, encephalitis, stroke, meningitis, Guillain-Barre syndrome, seizures, or unexplained neurological symptoms. This was an observational, mixed retrospective and prospective study. The objective was to define the neurological manifestations of MIS-A and identify their accompanying clinico-radiological and laboratory features, that aided in the diagnosis.

The following case definition outlined by the CDC was used to define MIS-A $A^{5}$;1) A severe illness requiring hospitalization in an adult $\geq 21$ years; 2) A positive test result for current or previous SARS-CoV-2 infection (nucleic acid, antigen, or antibody) during admission or in the previous 12 weeks; 3) Severe dysfunction of $\geq 1$ extrapulmonary organ systems (e.g., hypotension or shock, cardiac dysfunction, arterial or venous thrombosis or thromboembolism, or acute liver injury); 4) Laboratory evidence of severe inflammation (e.g., elevated CRP, ferritin, D-dimer, or interleukin-6); and 5) Absence of severe respiratory illness. Eight cases of MIS-A with primary, severe neurological manifestations were identified. Institutional review board approval was obtained.

\section{Case 1}

A woman in her 50's was admitted with unresponsiveness 6 days after recovery from COVID-19. Magnetic resonance imaging (MRI) showed multiple thalamic, cerebellar and left posterior cerebral artery territory infarctions. She was started on enoxaparin, Aspirin, and Levetiracetam. Her inflammatory markers were elevated (Table). On examination she was drowsy, febrile, had tachycardia, dense right hemiplegia, and bilateral extensor plantar responses. Multi-organ dysfunction was also identified. MR angiogram was normal. Echocardiogram and holter monitoring were normal. She was started on IV methylprednisolone $1 \mathrm{gm} /$ day for 5 days. Over the next few days, her platelet counts dropped to a nadir of $30 \mathrm{~K} / \mathrm{uL}$ and she was started on plasmapheresis, and IVIg. She was transferred for rehabilitation after 2 months with residual aphasia and right hemiparesis. 


\section{Case 2}

A man in his 60's was admitted with acute right hemiplegia and aphasia (NIHSS score 24). At admission SARS-CoV2 RT-PCR was negative. He had a prior history of COVID-19 illness 6 months earlier. He had received 1 dose of inactivated COVID vaccine (COVAXIN ${ }^{\mathrm{TM}}$, Bharat Biotech) 1 month earlier (partially vaccinated status). CT angiogram (CTA) showed a left internal carotid artery (ICA) and left PCA occlusion. He was administered IV Tenecteplase. The next day, CT brain showed large left midbrain, thalamic and occipital infarcts. On day 3 he developed fever and tachycardia. Inflammatory parameters were elevated (Table). On day 4, he was intubated for an MRI which showed new infarcts in the left middle cerebral artery (MCA) territory and right frontal area. On day 5, bronchoalveolar lavage obtained at the time of intubation was positive for SARS-CoV2 RT-PCR. He was diagnosed with post-vaccination (PV), COVID reinfection associated MIS-A [PV-MIS-A]. CSF showed mild pleocytosis and was negative for SARS-CoV2 RT-PCR. He stabilized with IVMP.

\section{Case 3}

A man in his late 20's was admitted with chest pain, myalgia, fever, and vomiting, 8 weeks after COVID19. On examination, he was febrile $\left(39.5^{\circ} \mathrm{c}\right)$, tachycardic, and tachypneic. Evaluation revealed myocarditis, pericarditis, hepatitis, and acute kidney injury. One week later, he developed difficulty in walking, urinary hesitancy, and paraesthesia in both feet. Examination revealed spasticity in his lower limbs (LL), reduced motor strength [2/5 in both LL], bilateral knee synovitis, exaggerated deep tendon reflexes and reduced touch sensations till the knees. MRI brain was normal but the spinal cord showed hyperintensities involving the lower thoracic cord and conus. (Fig. 1) He was treated with 3 days of IVMP and completely recovery within 3 days.

\section{Case 4}

A woman in her 60's presented with a large left MCA stroke. Mechanical thrombectomy was attempted with unsuccessful recanalization. SARS-CoV2 PCR was positive on day 2. She required vasopressor support for the next few days but slowly stabilized. At follow up 5 months later, she was aphasic (mRS4). (Table 1)

\section{Case 5}

A man in his 40's presented with fever and unresponsiveness for 1 day. Evaluation revealed LVO. After admission he developed rhabdomyolysis, and acute kidney injury over the next 2 weeks and succumbed to multi-organ dysfunction. (Table 1).

\section{Case 6}


A man in his late 30's presented with recurrent fever after a 4 day asymptomatic period. 2 days later, he developed a right hemiplegia and aphasia. He was brought 15 hours after the onset and mechanical thrombectomy was attempted. In spite of $\mathrm{TICl} 2$ a revascularization, he worsened and developed complications such as an orbital infarction syndrome and worsening midline shift requiring decompressive craniectomy.

\section{Case 7}

A man in his 40's presented to the hospital with fever and right sided weakness. His inflammatory markers were elevated and CT brain showed subcortical hypodensities with gyral enhancement. SARSCoV2 RT PCR was positive on day 3. MRI brain also showed striking gyral enhancement. CSF examination was normal and CSF SARS-CoV2 RTPCR was negative. COVID encephalitis was considered. He improved within 15 days of treatment with Dexamethasone, and antiplatelets.

\section{Case 8}

A woman in her 30's presented to us with fever, altered mental status and a tender mass at the angle of the left jaw. She had a history of COVID-19, 20 days earlier, which had been treated at home. On examination, she was delirious, febrile, tachycardic and had neck stiffness and diffuse cervical lymphadenopathy. MRI brain and CSF examination were normal. Echocardiogram and cardiac biomarkers were normal. She was started on antibiotics and IV methylprednisolone $1 \mathrm{gm} /$ day. She improved on day 2, but developed acute left ventricular failure and cardiogenic shock on day 3. After intubation, mechanical ventilation and inotropic support, she went on to develop atrial fibrillation, acute renal shutdown and acute liver failure, rhabdomyolysis and Critical illness myo-neuropathy by day 5 . She was treated with SLED (slow low efficiency dialysis) and IVIg $2 \mathrm{gm} / \mathrm{kg}$ over 5 days. She is still hospitalized with an MRC sum score of 4 and on ventilatory support after 25 days.

\section{Results}

Over a 12-month period, eight patients (2.2\%) admitted to our ICUs had a predominant neurological MISA, where the admitting diagnosis was a severe neurological condition and primary neurological care was delivered. The male: female ratio was 5:3 and the average age was 44.5 (range 33-62). Five patients had no previous co-morbidities (62.5\%), two were hypertensive and one was a smoker. Most patients were unvaccinated (75\%). Two patients developed COVID-19 after partially vaccination with inactivated vaccines (Single dose) with Covaxin ${ }^{\mathrm{TM}}$ (BBV152) [ Bharat Biotech] and Sinopharm COVID-19 vaccine ${ }^{\mathrm{TM}}$ [BBIBP-CorV] (Sinopharm's Beijing Institute of Biological Product). The patient who received Covaxin ${ }^{\mathrm{T}}$ (BBV152) had a concurrent COVID-19 reinfection during his MIS-A. Only 50\% of patients (4/8) had severe cardiac involvement requiring inotropic support [Myo-pericarditis, cardiogenic shock or hypotension requiring vasopressors] and evidence of acute liver injury. $37 \%$ of patients had an acute kidney injury (3/8), $25 \%$ had dermatological manifestations (2/8). $75 \%$ of patients had lymphopenia (ALC < 1000 / $\mathrm{cmm}^{3}$ ). Elevated thrombo-inflammatory parameters included D-Dimer $-6328 \mathrm{ng} / \mathrm{ml}$ (range 960-18060) 
[Normal $<500 \mathrm{ng} / \mathrm{ml}$ ] and serum ferritin 1218 (range 400-1832) [normal - 20-250 ng/ml]. D-dimer levels were elevated above 5 times the upper limit of normal in $75 \%(6 / 8)$.

CRP was elevated in $100 \%$ with an average range of 119 (32-280), platelet counts were 397,000 (range 30-900 x K/uL), One patient developed severe pancytopenia with thrombocytopenia ( $30 \mathrm{~K} / \mathrm{uL}$ ), whereas one patient developed severe asymptomatic thrombocytosis $(900 \mathrm{~K} / \mathrm{uL}) .75 \%(6 / 8)$ required mechanical ventilatory support, IVIG was administered in $25 \%$ (2/8), IVMP in $7 / 8$ ( $87.5 \%)$, Plasmapheresis in $12.5 \%$ $(1 / 8)$

The neurological spectrum comprised of stroke with large vessel occlusion (LVO) in 4 patients (50\%), embolic stroke in 1 patient (12.5\%), transverse myelitis (12.5\%), COVID encephalitis (12.5\%), rhabdomyolysis in 2 patients (25\%), acute encephalopathy (12.5\%) and critical illness myo-neuropathy (12.5\%). LVO could not be fully recanalized in one patient because of extreme vessel tortuosity and only partial recanalization was obtained in the other because of clot length. Other factors in our cohort included late presentation to hospital and infarction in a different territory from the LVO observed in the CTA. The mean time to diagnosis of MIS-A was 4.5 days (ranging from 1-7 days).

Six out of eight patients had significant residual neurological impairment (mRS 4-6). Only two patients improved or recovered completely.

\section{Discussion}

MIS-A and MIS-C are heterogeneous disorders with a variable clinical spectrum. Delayed diagnosis, increasing severity of illness, and severe neurological involvement increase mortality and morbidity. Neurological manifestations are often overshadowed by cardiac or multi-organ dysfunction, and are reported only in around $23 \%$ of MIS-C. ${ }^{21}$ Organ symptoms that are prominently involve the gastrointestinal and cardiac systems.

Our series demonstrates prominent and early neurological manifestations of MIS-A. $67.5 \%$ of patients experienced disabling strokes, however transverse myelitis COVID encephalitis, rhabdomyolysis, acute encephalopathy and critical illness myo-neuropathy (CIMN) were also encountered. Importantly $50 \%$ did not have a significant cardiac illness. When severe cardiac manifestations developed, they occurred later in the illness leading to a delayed consideration of MIS-A. ${ }^{1}$

The average time to recognition of MIS-A was longer in patients without early cardiac manifestations (4.1 days) as compared to patients with early cardiac manifestations (1.5 days). However, this discrepancy is also explained by the earlier consideration of MIS-A when cardiogenic manifestations supervened as well as increasing familiarity with MIS-A during the later part of the study.

D-dimer, CRP, ferritin were the most frequently elevated laboratory markers. Lymphopenia was frequently encountered and the median number of organ systems involved was 3.37 (range 1-5). 
Anticipating that MIS-A may be reported as an adverse event following immunization (AEFI), three postvaccination (PV-AEFI) scenarios have been postulated. ${ }^{6}$ [Figure 3] In our cohort, we encountered a fourth scenario: MIS-A in two partially vaccinated patients with concurrent COVID-19 re-infection in one and new COVID-19 infection in the other (10 days after the first dose). (Fig. 3). Whether the MIS-A was precipitated by SARS-CoV-2 infection or potentiated by immunization is unclear (as in antibody dependent enhancement). Nevertheless, the first case (Patient no 2) developed MIS-A within the expected timeline for a PV-MIS-A (> 4 weeks). It was also difficult to differentiate between COVID-19 associated encephalitis or a MISA-encephalitis.

COVID-19 induces both a thrombocytopathy (functional platelet disorders affecting either platelet activity and/or function) and an endotheliopathy (functional endothelial disorders affecting either endothelial activity and/or function). The combination of these two leads to a thrombo-inflammation. ${ }^{7}$ The SARS CoV-2 virus also impairs the immune system resulting in a $T_{h} 2$ response against the virus rather than the essential $T_{h} 1$ response. This excessive humoral response with a diminished cellular immune response can lead to severe disease manifestations as well as a Type 3 hypersensitivity with deposition of immune complexes in blood vessels, complement activation and recruitment of other immune cells such as, producing a COVID microvasculitis. ${ }^{8}$

Although strokes are uncommon in COVID-19 and account for $<1.3 \%$ of complications, they are more disabling and associated with higher mortality. ${ }^{9}$ Multiple mechanisms are responsible for ischemic strokes in COVID-19. While the main mechanisms include hypercoagulability, vasculitis, thromboinflammation and cardiomyopathy. Secondary hemorrhagic transformation of ischemic strokes may be due to endothelial damage or a COVID-19 associated consumption coagulopathy. ${ }^{10}$

ACE 2 receptors have been found in the diaphragm of some patients with COVID-19. ${ }^{11}$ This may have a role in prolonged respiratory muscle weakness and longer duration of mechanical ventilation. Critical illness myopathy (CIM) and polyneuropathy (CIP) are found in patients with severe COVID-19 after prolonged ICU stay. Other factors such as medications, steroids, critical illness per se, muscle hypoperfusion and metabolic factors also contribute to CIM and CIP. ${ }^{12,13}$ Almost $60 \%$ of those who die during acute COVID-19 show muscle inflammation, ranging from mild to severe inflammatory myopathy. In fact, SARS-CoV-2 is thought to be associated with an immune-mediated myopathy. ${ }^{14}$

Whether similar mechanisms are at play in MIS-A are as yet unknown. Nevertheless, the para or post infectious hyperinflammatory host response is likely to injure the same structures as in acute COVID-19. Thus, the neurological manifestations of MIS-A mirror those seen in acute COVID-19, albeit with a lower frequency due to the rarity of this syndrome compared to the pandemic.

As MIS-A is still a rare condition and our sample size is comparatively small, we could have missed other neurological manifestations of MIS-A. Nevertheless, as our study was conducted in a quaternary care hospital, where complex cases are admitted, our study findings are likely to be generalisable to the population. 
In conclusion, conspicuous neurological manifestations with MIS-A are rare $(>2.2 \%)$ and often overshadowed by multi-system organ dysfunction. However, neurological disorders can be the presenting manifestation of a MIS-A. Such patients also may be left with greater morbidity and mortality.

- The STROBE check list for observational studies has been followed.

\section{Declarations}

We have obtained the necessary Ethics committee approval from our institute Patient consent has been obtained in all the cases mentioned in the article

\section{Funding}

None

\section{Disclosures}

None

\section{References}

1. Davogustto GE, Clark DE, Hardison E et al. Characteristics Associated With Multisystem Inflammatory Syndrome Among Adults With SARS-CoV-2 Infection. JAMA Network Open 2021;4(5)

2. Nepal G, Shrestha GS, Rehrig JH et al. Neurological Manifestations of COVID-19 Associated Multisystem Inflammatory Syndrome in Children: A Systematic Review and Meta-analysis. Journal of Nepal Health Research Council 2021;19(1)

3. Abel D, Shen MY, Abid Z et al. Encephalopathy and bilateral thalamic lesions in a child with MIS-C associated with COVID-19. Neurology 2020;95(16)

4. Mavrogeni SI, Kolovou G, Tsirimpis V, Kafetzis D, Tsolas G, Fotis L. The importance of heart and brain imaging in children and adolescents with Multisystem Inflammatory Syndrome in Children (MIS-C). Rheumatology International 2021;41(6)

5. Morris SB, Schwartz NG, Patel P et al. Case Series of Multisystem Inflammatory Syndrome in Adults Associated with SARS-CoV-2 Infection - United Kingdom and United States, March-August 2020. MMWR Morbidity and Mortality Weekly Report 2020;69(40)

6. Vogel TP, Top KA, Karatzios C, et al. Multisystem inflammatory syndrome in children and adults (MIS$\mathrm{C} / \mathrm{A})$ : Case definition \& guidelines for data collection, analysis, and presentation of immunization safety data. Vaccine $2021 ; 39(22)$.

7. Gu SX, Tyagi T, Jain K et al. Thrombocytopathy and endotheliopathy: crucial contributors to COVID19 thromboinflammation. Nature Reviews Cardiology 2021;18(3) 
8. Roncati L, Nasillo V, Lusenti B, Riva G. Signals of Th2 immune response from COVID-19 patients requiring intensive care. Annals of Hematology 2020;99(6)

9. Qureshi Al, Baskett WI, Huang W et al. Acute Ischemic Stroke and COVID-19. Stroke 2021;52(3)

10. Spence JD, de Freitas GR, Pettigrew LC et al. Mechanisms of Stroke in COVID-19. Cerebrovascular Diseases 2020;49(4)

11. Shi Z, de Vries HJ, Vlaar APJ et al. Diaphragm Pathology in Critically III Patients With COVID-19 and Postmortem Findings From 3 Medical Centers. JAMA Internal Medicine 2021;181(1)

12. Maramattom BV, Wijdicks EFM. Neuromuscular Disorders in Medical and Surgical ICUs: Case Studies in Critical Care Neurology. Neurologic Clinics 2006;24(2)

13. Maramattom BV, Wijdicks EFM. Acute neuromuscular weakness in the intensive care unit. Critical Care Medicine 2006;34(11)

14. Aschman T, Schneider J, Greuel S et al. Association Between SARS-CoV-2 Infection and ImmuneMediated Myopathy in Patients Who Have Died. JAMA Neurology 2021

\section{Table}

Table 1 is available in the Supplementary Files.

\section{Figures}
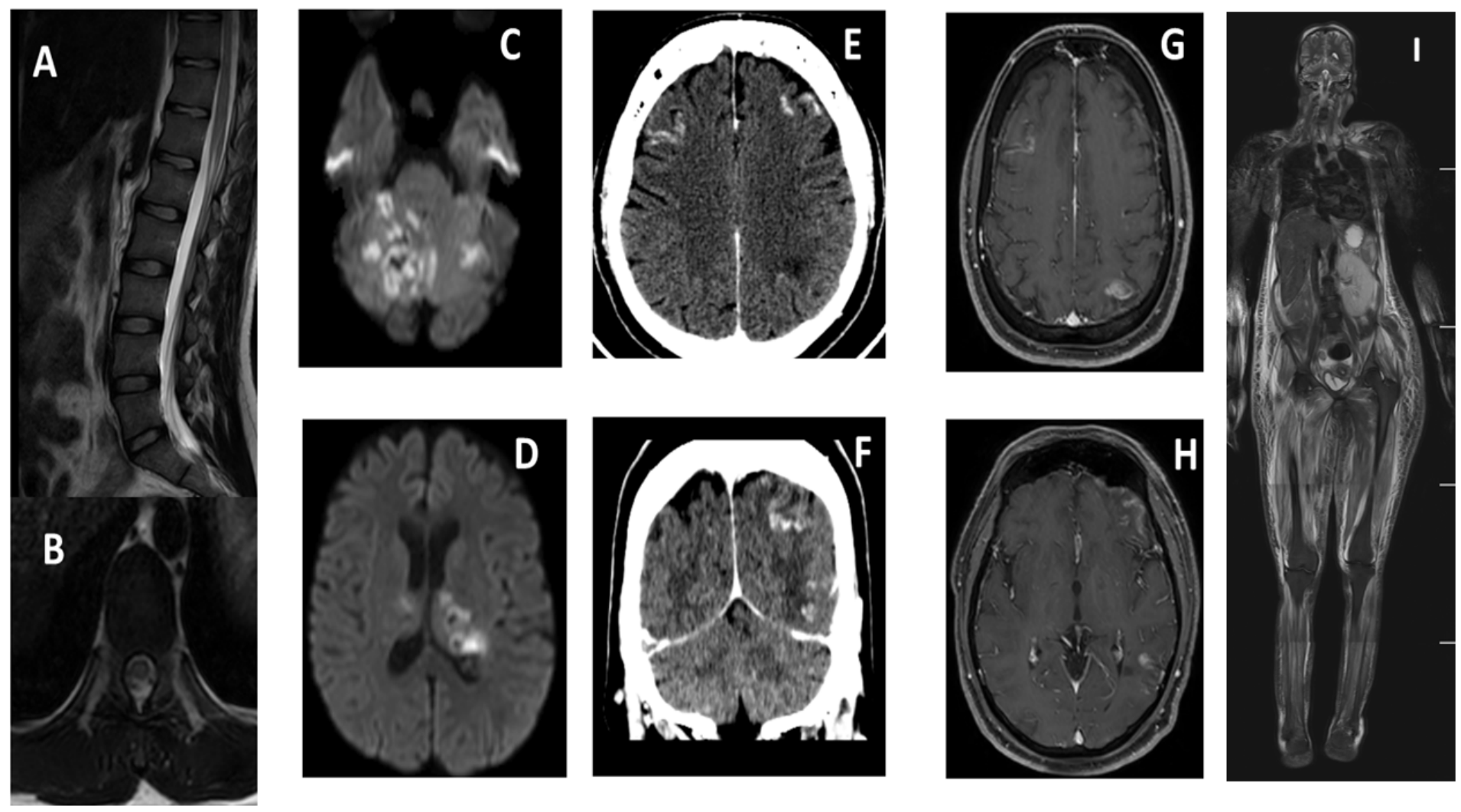

Figure 1 
MRI images. Panel A and B: Sagittal and axial T2 weighted images of the lower spinal cord showing a subtle thoraco-lumbar cord hyperintensity. Panel C and D; Diffusion weighted MRI images showing scattered cerebellar and thalamic infarctions. Panel E and F; CT brain images showing cortical enhancement in Case 7. Panel $\mathrm{G}$ and $\mathrm{H}$. T1 post contrast axial images showing striking gyral contrast enhancement in Case 7. Panel I- Whole body STIR muscle MR imaging diffuse hyperintensity involving the muscles and soft tissue of both upper limbs, neck, shoulder girdle, paraspinal region, chest wall, abdominal wall, pelvis and both lower limbs. Diffuse subcutaneous edema is noted in both lower limbs.
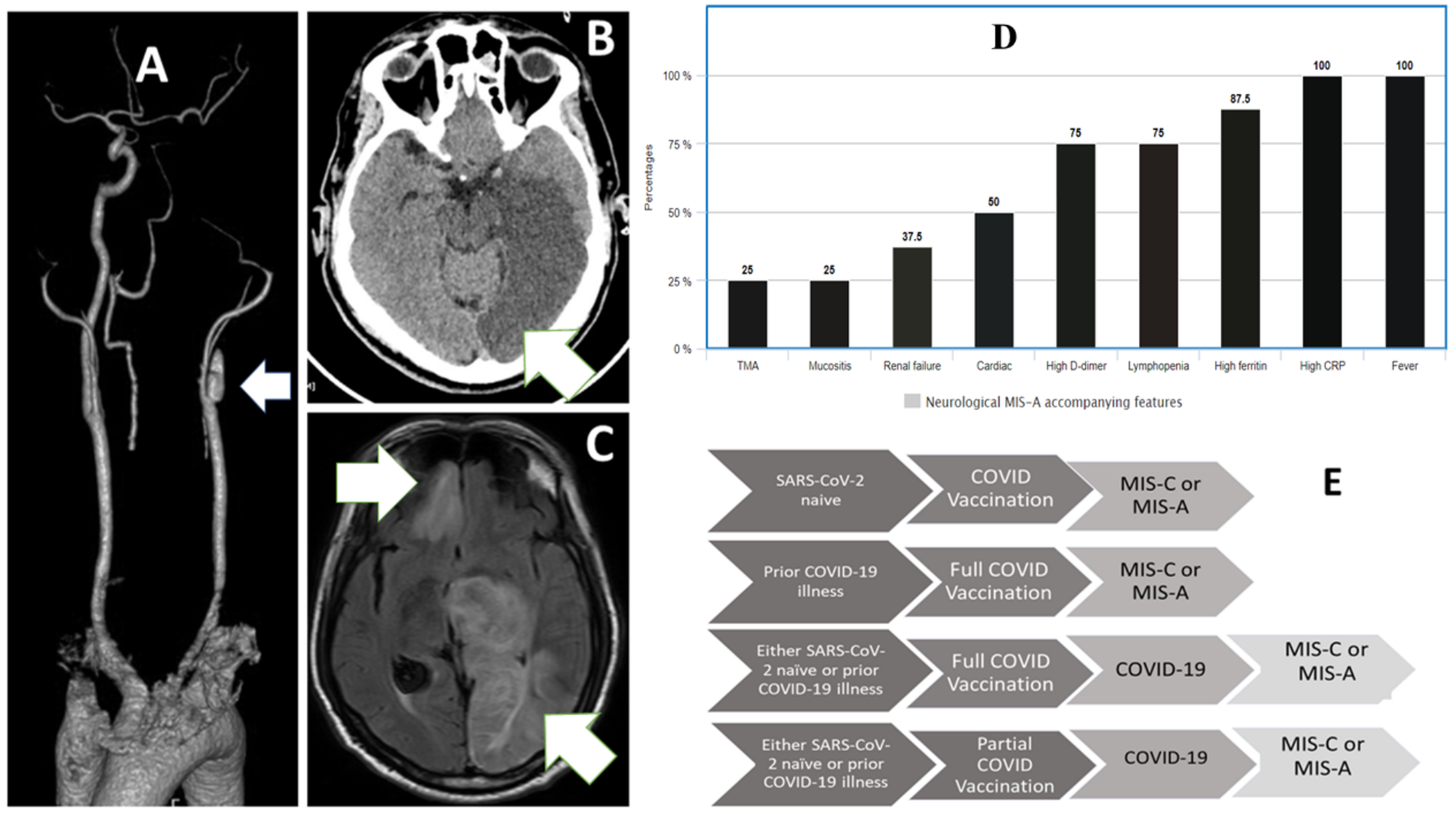

Figure 2

Panel A: CT angiogram shows a left ICA occlusion at the origin (White arrow). Panel B: CT brain on day 2 showing a left PCA territory infarction. Panel C: FLAIR MRI on day 5 showing a left thalamic, and occipital infarction along with a right frontal infarct. Panel D: Frequency of different clinical and laboratory manifestations in our cohort. Panel E panel: Post vaccination MIS-A scenarios. The bottom scenario shows the PV- MIS-A scenario in our patient.

\section{Supplementary Files}

This is a list of supplementary files associated with this preprint. Click to download.

- MISAtableNCS1.docx

- STROBEMISA.doc 